\section{La resiliencia: un factor decisivo para el crecimiento y mejora de las organizaciones}

\author{
Resilience: decisive factor for growth and improvement of \\ organizations
}

\begin{abstract}
RESUMEN
El término de globalización, según algunos estudiosos, aparece y comienza a ser utilizado en 1771 y la consecuente difusión hace que llegue a diferentes ámbitos y hoy, con más frecuencia y circunscrito a las empresas, donde estas tienen la necesidad de primero a desaprender para luego aprender; es decir, a desaprender la forma de hacer y toma decisiones de manera tradicional de trabajo, con la única finalidad de permanecer en este mercado que es altamente competitivo. Sin embargo, en la medida que las empresas continúen realizando cambios, existe la posibilidad de que se intensifiquen los problemas en el trabajo y, por ende, se genere un clima organizacional inadecuado. En ese sentido, el presente artículo plantea la importancia que tiene la resiliencia en las empresas, ya que su aplicación contribuye a mejorar la capacidad de adaptarse de manera exitosa y de enseñar el camino adecuado hacia el progreso y no solo a su supervivencia.
\end{abstract}

Palabras claves: Resiliencia; globalización; clima organizacional.

\section{ABSTRACT}

The term of globalization according to some scholars appears and begins to be used in 1771 and the consequent diffusion makes it arrive at different scopes and today more frequently and circumscribed to the companies, where they have the necessity of first to unlearn and then to learn; That is, to unlearn their traditional ways of making and making decisions, with the sole purpose of remaining in this market that is highly competitive. However, as companies continue to make changes, there is a possibility that work problems will intensify and thus create an inadequate organizational climate. In this sense, the present article raises the importance of the resilience in the companies, since its application contributes to improve the capacity to adapt successfully and to teach the proper way to the progress and not only to its survival.

Keywords: Resilience; globalization; organizational climate.

\section{Giovanna Serna Silva}

gia.silvass@gmail.com

\section{Carmen Zenozain Cordero}

carmenzenozain@yahoo.com

Johannes Schmidt Urdanivia

unmsmfca@gmail.com

Universidad Nacional Mayor de San Marcos, Facultad de Ciencias Administrativas

(C) Los autores. Este artículo es publicado por Gestión en el Tercer Milenio de la Facultad de Ciencias Administrativas de la Universidad Nacional Mayor de San Marcos. Este es un artículo de acceso abierto, distribuido bajo los términos de la licencia Creative Commons Atribucion - No Comercia_Compartir Igual 4.0 Internacional. (http://creativecommons.org/licenses/by-nc-sa/4.0/) que permite el uso no comercial, distribución y reproducción en cualquier medio, siempre que la obra original sea debidamente citada. 


\section{INTRODUCCIÓN}

\section{Conceptualización en términos generales:}

Tal vez, para muchos de nosotros, el término de resiliencia no es muy común o tal vez lo estamos escuchando por primera vez, pero en realidad ¿qué significa la Resiliencia?

El concepto Resiliencia, se origina del latín, de la palabra "Resilio" da un sentido de regresar atrás, retroceder de un salto, resaltar o rebotar (Becoña, 2006). Como manifestamos este término, un tanto antiguo, sigue vigente y con más fuerza en la actualidad; además este concepto es ampliamente utilizado en muchos ámbitos desde su aplicación en el individuo como ser, así como en las organizaciones empresariales y ha ayudado a dar explicación de eventos que han ido brotando en la vida de estos individuos y, más tarde, en la vida de las organizaciones. Veamos algunas definiciones acuñados por diferentes autores:

Rutter (1972), fue el primero que acuñó el término resiliencia, para hacer referencia a las ciencias sociales y lo conceptualiza de la siguiente manera: "La resiliencia se comprende como la variación individual en la manera en la que las personas responden a los riesgos a lo largo del tiempo".Asimismo, afirma que "los perfiles resilientes están presentes tanto en el individuo como en el contexto ambiental", poniéndose de manifiesto en las situaciones más desfavorables, y que la mayoría de los individuos están propensos o vulnerables a enfrentarse a contingencias.

En Psicología se dice que es la "Habilidad para resurgir de la adversidad, adaptarse, recuperarse y acceder a una vida significativa y productiva", teniendo en cuenta que la habilidad es la capacidad o maestría de una persona para realizar una actividad correctamente y con facilidad. Es la destreza para realizar algo que se da desde que nacemos, o se adquiere y perfecciona, gracias al aprendizaje y la práctica que desarrollemos. Además, es interesante que estas habilidades del sujeto estén muchas veces no exploradas y es necesario ponerlas al descubierto. No consiste en evitar el riesgo, sino que el organismo, el ser como tal, supere con éxito las diferentes pruebas.
La resiliencia distingue componentes:la primera, la resistencia frente a situaciones de destrucción, de desolación; es decir, la capacidad de protegerse a uno mismo bajo presión y, por otra parte, el que va más allá de la resistencia, la capacidad de resolverse a tener un comportamiento trascendente $\mathrm{y}$ positivo, pese a las circunstancias difíciles. (Vanistendael,1994)manejándose a pesar de las dificultades. Y un tercer componente: el aprendizaje y lección para enfrentar a otras situaciones, sea similares o diferentes. Un punto a tomar en cuenta es que estos hechos o eventos de crisis, no se presentan de manera automática o determinada; es decir, a intervalos como edad, sexo, geografía o status socioeconómico, sino que se da en cualquier período o circunstancia en la vida de un individuo.

Asimismo,Grotberg (1995) en esta misma línea, aduce que es la "Capacidad del ser humano para hacer frente a las adversidades de la vida, superarlas, $e$, inclusive, ser transformada por ellas". Y más tarde, Luthard y Cicchetti (2000) la conceptualiza como el "Proceso dinámico que tiene como resultado la adaptación positiva en contextos de gran adversidad". Por lo tanto, la resiliencia es la capacidad que tienen los individuos frente a las adversidades, infortunios para mantenerse en pie de lucha; con dosis de perseverancia, tenacidad, actitud positiva y acciones, que permiten avanzar en contra de la corriente y superarlas Chávez, E. y Yturralde, E.(2006).

También, como una organización científica de psicólogos que tiene como función la promoción de la salud ha considerado a la resiliencia como "el proceso de adaptarse bien a la adversidad, a un trauma, tragedia, amenaza, o fuentes de tensión significativas, como problemas familiares o de relaciones personales, problemas serios de salud o situaciones estresantes del trabajo o financieras. Significa "rebotar" de una experiencia difícil, como si uno fuera una bola o un resorte"; por lo tanto, frente a estas circunstancias es necesario una actitud positiva $^{1}$ (aprender a pensar en positivo), y proactiva $^{2}$.

1 La actitud positivapuede definirse como la disposición o el ánimo con el que enfrentamos una situación. Por tanto, se deduce que una actitud positiva es aquella que adoptamos para afrontar lo que venga de la forma más beneficiosa para nosotros. https:// tusbuenosmomentos.com/que-es-la-actitud-positiva/ 


\section{La resiliencia en las organizaciones:}

Las habilidades, que poseen los individuos para salir airosos de problemas que surgen muchas veces en forma insospechada, todo esto con un plus que es el de salir fortalecidos, con una actitud de lucha y aprendizaje, fue introducido y aplicado para el caso de las empresas con el objeto de crear los contextos para que estas utilicen semejantes principios, elementos y fortalezas en solucionar sus conflictos, tanto internos como externos. Siendo así, veamos a algunos autores que toman el contexto organizacional para ensayar definiciones que podemos utilizarlas en este acápite.

Por tal hecho, este término siendo aplicable al comportamiento de personas, sus reacciones y su capacidad de enfrentarlas, se puede también aplicar al entorno organizacional y la podemos definir como al beneficio que tienen las empresas al adecuarse al cambio; enfocándose principalmente en adaptaciones positivas y de aprendizaje ante situaciones adversas, que se puedan presentar tanto en lo interno y externo de las organizaciones. "Asimismo permite que las organizaciones puedan esquivar tendencias a las que no se pueden ajustar y poder tener las herramientas necesarias para enfrentar 10 inesperado" (Rodríguez, 2016).

Kotliarenco, I. (2011) señala que, "la resiliencia es un proceso dinámico, constructivo, de origen interactivo, sociocultural que conduce a la optimización de los recursos humanos y permite sobreponerse a las situaciones adversas. Se manifiesta en distintos niveles del desarrollo, biológico, neurofisiológico y endocrino en respuesta a los estímulos ambientales", por lo que creemos que este proceso dinámico es una selección de aquellos componentes que contribuyan a generar los modos de comportamiento y actuación más adecuados para luego determinar el tipo de acción a tomar, omitiendo toda clase de aspectos irrelevantes y que no estimulen progreso a la organización.

Entonces, es esta capacidad que tienen las empresas para planificar y adaptarse

2 UCA (Universidad de Cádiz), 2017. La proactividad es la actitud en la que la persona asume el pleno control de su conducta vital de modo activo, lo que implica la toma de iniciativa en el desarrollo de acciones creativas y audaces para generar mejoras, haciendo prevalecer la libertad de elección sobre las circunstancias de la vida. a los cambios, a las rupturas bruscas y a la desorganización a través de la anticipación, prevención, la protección, la capacidad de respuesta y la capacidad de recuperación Gonzales(2015), esto le permite mejorar tanto más transformarse llegando a aprender de todo lo sucedido y tomarlo ya no como un trauma, una tragedia o amenaza -como se vio-, sino más bien como una experiencia útil que le ayudará tomar nuevos bríos para enfrentarse a lo que vendrá después. Se trata de usar proactivamente la experiencia estresante, para obtener un beneficio que no estaba planeado; pero que en el momento se convierte en una oportunidad para crear soluciones de beneficio individual (persona o empresa) que posteriormente, al ser replicados pueden llegar a convertirse en modelos de desempeño grupal, aprovechado por la Gerencia de toma de decisiones reales positivas y justo a tiempo.

Una organización es resiliente cuando posee la capacidad $^{3}$ de resistir a la incertidumbre, de hacer frente a las crisis de manera exitosa, encararse a los cambios y situaciones conflictivas; como camino hacia el progreso y no solo como mecanismo de supervivencia. Además, es importante precisar que la disposición de afrontar estos momentos de inestabilidad, crisis y hasta incertidumbre puede simplemente liberar el potencial y el talento de toda la organización interna de la empresa; siendo muchas veces la principal causa de la creatividad y la innovación. Estos, utilizados como medios para crear recursos que permitan superar las inestabilidades que se afronta y las que se puedan presentar en un futuro, la cual no es muy lejano por los constantes cambios que se presenta en el día a día empresarialy, por otra parte la globalización mundial.

Medina (2012)expone que las empresas que están sometidas a una mayor resiliencia, desarrollan algunas características que las hacendistintas de otras empresas que no tuvieron este distintivo que las hacen Resilientes, y las mencionamos a continuación:

3 RAE. Real Academia Española.Capacidad, son los recursos $\mathrm{y}$ condiciones que tiene un individuo, una empresa, para desempeñar una determinada tarea o actividades, disposición o prudencia para resolver situaciones. 
1. Tienen un ethos organizacional de esfuerzo constante, orientado a generar una mayor resistencia a las catástrofes.

2. Presentan un buen conocimiento de la situación, de las amenazas y oportunidades enfrentadas por la organización, a través del seguimiento activo de las señales fuertes y débiles provenientes del contexto.

3. Poseen un fuerte compromiso encaminado a la identificación proactiva y la gestión de las vulnerabilidades clave.

4. Presentan una cultura promotora de sus capacidades de adaptación, agilidad e innovación dentro de la organización.

Cabe mencionar que estas características dependerán de cada empresa y la política empresarial, sabiendo que cada una presenta su propia tempestadde contingencias y su propia manera de resolverlas combinando experiencia, tiempo en el mercado, rubro y principalmente los valores compartidos dentro de la organización; la cual podrá sustentar su actuación y capacidad final frente a eventos críticos y salir triunfantes.

A la luz de lo anterior, a pesar que, muchas veces se relaciona y/o confunda dos términos que, para efectos de la presente publicación es necesario precisar la diferencia muy sutil que existe entre estas, las organizaciones resistentes con organizaciones resilientes. El primero, puede resistir cualquier tipo de presión o ataque sin deteriorarse o dar muestras de debilidad ${ }^{4}$; yen el segundo concepto, la organización no solo se limita a esquivar el temporal, sino que surge transformada positivamente por las experiencias de la adversidad y logra total, o en gran parte, salir triunfante de este cúmulo de circunstancias, momentos o tiempos malos; así como más posicionada en el mercado. Así mismo, reconstruirse, innovándose creativamente, convirtiendo los aspectos negativos en nuevas oportunidades de permanecer en el mercado y tomándolas como ventajas comparativas con el merado empresarial. El reto es entender la

\footnotetext{
4 Free Dictionary. Debilidad, que se muestra dominado o sin poder de decisión, por lo que muchas veces son objeto de manipulaciones o engaños, y, en las empresas son todas aquellas dificultades o carencias que una empresa posee y no le permite mejorar y avanzar ante su competencia..
}

dificultad como una invitación a dar lo mejor de nosotros mismos, teniendo presente que pueden ser afrontados solamente a través de la utilización de la creatividad, trabajo arduo, el compromiso con uno mismo y con la empresa y la innovaciónJericó (2009).

Según León, (2015), citando a Clegg / Porras, 2007 y a Nemeth, / Hollnagel, / Dekker, 2009, menciona que en la resiliencia organizacional se identifican tres etapas, las cuales veremos:

1) La prevención de acontecimientos negativos, que vuelven a las empresas menos vulnerables, tomando en cuenta su entorno que la hace más competitiva; generalmente es el factor que vuelve más vulnerable a las organizaciones, especialmente las vinculadas con actividades relacionadas a los procesos de globalización (Clegg / Porras, 2007).

2) Prepararse para prevenir que los resultados negativos no se agraven con el transcurrir del tiempo,mantenerlos un paso adelante mediante planes de acción inmediata evitando que se desplomen y desaparezcan del mercado. Porque detrás de nuestra empresa existen muchas otras intentado entrar en nuestra posición en el mercado.

3) Contar con la estructura adecuada y flexibleparaquedespuésdesalirvictoriosos, hagamos frente a las secuelas negativas que dejan esos acontecimientos;aplicando en forma dinámica, pero flexible, acciones que tienen como objetivo recuperar sus fortalezas y eficiencia después del trauma experimentado y la mala experiencia obtenida (Nemeth, / Hollnagel, /Dekker, 2009).

A raíz de esto, se plantea que la citada estructura se entienda como un conjunto de relaciones que mantienen entre sí las partes de un todo, que va más allá de lo tangible de la organización (activos corrientes y no corrientes). Implica el capital más importante de la organización: su gente, sus colaboradores (desde los diferentes niveles)a quienes se deberá prestar atención y dar una buena dosis de motivación al grado preciso. 


\section{Educar a las organizaciones en la resiliencia:}

Talcomo elserhumano necesita elapoyo paraque desarrollen una forma provechosa y ventajosa de enfrentarse a la vida; será necesario hacerlo mediante un aspecto psicológico-motivacional positivo, que logre una buena autoestima en el individuo y que esto se transformará en una buena capacidad de resiliencia y así estar preparados para que puedan hacer frente a las diversas situaciones que pudieran presentarse en su camino por la vida. Sobrellevándolas sin sufrir un daño profundo, ni heridas profundas que no puedan ser cicatrizadas y dejadas como "experiencias de vida"; así también las organizaciones precisan pasar por esto mismo pero en un sentido macro.

Este hecho hace que nos preguntemos si, ¿Se nace con resiliencia?, o según Arboix (2014), citando a Fernández, explica, primero, que "es una capacidad humana de asumir con flexibilidad situaciones límite...es decir, todo el mundo tiene la capacidad de resistir el golpe, sobreponerse y seguir adelante; segundo, con referencia a la pregunta planteada responde, todos contamos con la capacidad de sobreponernos". Por lo tanto, esa capacidad que posee el humano le hace comprender que debe manifestar actitudes comportamientos que lo diferencien y lo ayuden a sobrellevar la experiencia y a trabajaren ello y seguir de continuo. Siendo así, la resiliencia la tenemos todas las personas; no es una excepción de algunos con características sobresalientes ni sorprendentes, es innato en el ser humano y es una respuesta común y es parte de nuestro. Como tal, es una capacidad dotada al ser viviente para sobrevivir y ser quienes somos; como vemos aquí, solo se trata de ver cuán resilientes podemos ser y si hay algunos aspectos en los que podemos mejorar para serlos, lo cual nos trae a reflexión lo mencionado por la misma psicóloga clínica cuando responde al decir;

"La resiliencia está asociada a unas características como el autoconocimiento, la autoestima, la independencia, la capacidad para relacionarse, la iniciativa, el buen humor y la creatividad, entre otras, y todas ellas forman parte, en mayor o menor intensidad, de las personas que se trasforman con el transcurrir de la vida. Sin embargo, es posible entrenarlas y mejorarlas. Por tanto,se puede afirmar que la resiliencia nace, se hace y se puede aprender". (Arboix, 2014)

Por lo que, aumentando algunas otras características que creemos muy necesarias como la disciplina, autoconfianza, compromiso con uno mismo y con los demás, empatía, adaptabilidad, flexibilidad, fuerza, actitud positiva, manejo de las emociones (inteligencia emocional), sentimientos de esperanza, humor; y porque no, la ayuda de un especialista, pueden ayudar la recuperación tanto físico-psicológica ${ }^{5}$ del individuo y de las organizaciones.

En el caso de las organizaciones es importanteque se establezca una política de aprendizaje, mantener prácticas saludables de adaptabilidad, compromiso de las diferentes áreas de la organización (promovida especialmente desde la alta dirección), disciplina. Asimismo, es un hecho que cuando la organización favorece al colaborador a actuar con resiliencia, ellos aprenderán a ver que los problemas, errores y fracasos son parte de la vida, aprenderán a desarrollar un sentido responsabilidad y manejo de eventos que los capacita para responder positivamente ante circunstancias con perseverancia. Se sabrá que siempre hay oportunidades que pueden ser aprovechables, flexibilidad y adaptables en el uso de los recursos empresariales manejando las herramientas a su alcance, potenciándolas de la mejor manera posible, y logrando una capacidad de recuperación aun lenta en muchos casos, pero posible.

Otro de los aspectos a considerar es la práctica de la cultura de la seguridad psicológica, que consiste en que los miembros de la organización expresen sus dudas, opiniones (favorables o contradictorias al de la Dirección, siempre en el rango del respeto), miedo de ser despedidos, miedo a ser reprendidos o a represalias; así como a no ser tomados en cuenta, trabajo excesivo. Además es importante

\footnotetext{
5 García, A. (2012)consecuencias físico - psicológicas son agresividad, terror, ansiedad, depresión, inseguridad, sentimientos de soledad, perdida y abandono, rudeza, explosiones de ira y violencia, trastornos de conducta, temerosos, alienados, dependencia, depresión, tristeza, ansiedad, dificultades en la comunicación con los demás, en las relaciones interpersonales y sociales pérdida de la autoestima, sentimientos de inferioridad, sentimientos de odio, trastornos del sueño, etc.
} 
que la organización tenga como política, la retroalimentación y reuniones constantes, fijación de metas organizacionales plasmadas con objetivos claros y precisos; políticas en baja rotación de los puestos de trabajo creando, de esta manera, vínculos de pertenencia y de "familia" entre los colaboradores. Se promueve el liderazgo y el trabajo en equipo, planteando nuevas oportunidades $\mathrm{y}$ formas de hacer las cosas, desarrollando un pensamiento crítico-analítico, capacidad de comunicación y resolución de problemas, y un constante espíritu de aprendizaje.

\section{El ubuntu: una actitud mental}

Por lo visto, tanto en los individuos y las-en el caso de las empresas también es importante, a la luz de estas declaraciones, que exista una iniciativa de la gerencia de poder ayudar al talento humano; dado que está formada por individuos que nacen con esa capacidad $y$, que mejor aún, pueden aprenderlas; enseñarles a interrelacionarse, enseñar a integrarse a la organización, recurrir al buen humor, a la creatividad y a un término que parece que sería muy interesante aplicar e insertar a este tema, el cual es el Ubuntu ${ }^{6}$ Este es un término referido a una actitud mental, enseñando, entre otros aspectos, que una persona llega a ser esta a causa de los demás, por los demás y para los demás; sobre todo, cuando es abierta y está disponible para los demás, respalda a los demás, no se siente amenazado cuando otros son capaces y son buenos en algo, porque está seguro de sí mismo ya que sabe que pertenece a una gran totalidad -a una familia empresarial-, quemengua cuando otras personas son avergonzadas o despreciadas, cuando otros son torturados u oprimidos - en caso de crisis. Todo ello confirma que su bienestar mental de pertenencia, compromiso, confianza y seguridad eleva la autoestima de las personas, por ello podemos concluir que los miembros de la organización estarán más preparadas para afrontar positivamente los cambios o lo que pueda acontecer en la vida empresarial, siendo este una filosofía que se puede adoptar en toda la organización encabezada por la Dirección hacia toda la organización.

6 UBUNTU:proviene de las lenguas zulúes y xhosa. Una palabra puede significar mucho, es una expresión mental, cuyo origen tiene de los indígenas al sur de África, que se propone la igualdad, la lealtad y solidaridad hacia los demás.
Un hecho que se debe tener en cuenta es que los individuos deben pasar por un proceso de asimilación y reflexión para salir de aquello que los perturbó Barrajón (2012); además, ante escenarios de desastre (natural o provocada por el ser humano) se debe considerar la formación de las llamadas «comunidades resilientes» cuyas características consisten en ser miembros de comunidades equivalentes o disímiles al mismo tiempo y, de alguna manera, estar vinculados a cada una de ellas por diferentes elementos, como la ubicación geográfica, su quehacer laboral, posición económica, género, intereses comunes. Existe un hecho común en estas comunidades que actúan interactivamente para cumplir algunas metas: seguir como grupo; después, de ello terminar y separarse al final. Así pues, se trata de una dinámica en la cual se podrían señalar las siguientes etapas que Ramos, R. 2014 señala que son:
El equilibrio que enfrenta a la tensión
El compromiso y el desafío

La superación

La significación y valoración

La positividad de sí mismo

La responsabilización

La creatividad

Como vemos, estas etapas son necesarias para poder superar mas no olvidar, son lecciones de aprendizaje y que nos ayudan a ser mejores como individuos y como miembros de una organización. Cada una de estas etapas son significativas y creemos necesaria hacer algún aporte respecto al punto seis sobre la responsabilización, entendida como "la exigencia impuesta por el principal a su agente, de que rinda cuentas por lo realizado en el marco de un contrato (explícito o implícito) que los vincula; o sea, es el acto o efecto de hacer responsable a alguien por los resultados, frente a los compromisos asumidos" Oszlak, O. (2003). Frente a la responsabilidad que se considera como una cualidad y un valor del ser humano, se puede ver como la sabiduría de entender que 
todo lo que hacemos o dejamos de hacer recaerá sobre nosotros mismos o sobre los demás, por lo que debemos enfocarnos en hacer bien las cosas. Asimismo, con la responsabilidad, el individuo aprende a actuar de manera que puedan confiar en él, ser un individuo confiable ya que esta garantiza la consecución de las responsabilidades adquiridas y genera un ambiente de seguridad y tranquilidad entre las personas, un capital emocional para la organización. Además, tengamos en cuenta que se necesita de un conjunto de acciones que puedan contrarrestar, efectivamente contra las situaciones nocivas y de crisis Flores y Grané (2008).

Otros procesos usados para promover la resiliencia en la organización son elestablecimiento de eventos o actividades y/o tareas de entrenamiento en resiliencia y manejo de estrés para lidiar con contextos estresantes que, a causa del trabajo y accionar de las empresas, sea por las tipologías del puesto o rol de trabajo, obstaculizan o menguan la capacidad del colaborador. Se busca, externamente, la realización de esta tarea; además, se recomienda que se efectúe una búsqueda y ayuda adecuados que permitan constatar el nivel y la "llegada" de los programas o talleres de ayuda a los trabajadores y cómo reaccionan ante los programas promovidos por la organización (Talavera, B. / Luceño, L. / García, J. / López, Mํㅡㄴ. 2012).

Los programas de resiliencia no solo son importantes, a nivel general u organizacional, sino también en los mismos puestos de trabajo; en las interrelaciones laborales, en casos de cese de trabajo, sino que además en circunstancias determinadas como el de confrontar una destitución, lidiar con las consecuencias de un incidente profesional, un enfrentamiento con otro colega, malos entendidos, accidentes, etc.

\section{El síndrome de burnout y el mobbing:}

Otro aspecto que según Talavera, B.,(et al)., puede considerarse a aquellos problemas que provocan situaciones perjudiciales $\mathrm{y}$ dañinas que surgen de forma sospechosa y se empeoran y aumentan a lo largo del tiempo, como el síndrome de burnout y el mobbing que pueden producir perjuicios para la salud del trabajador y desencadenar un gran número de demandas judiciales: Esto lleva a que las personas presenten altos niveles de resiliencia para hacerles frente.

El primero, el síndrome de burnout, es causado principalmente por la existencia de la gran competencia e incertidumbre laboral al que se ven sometidos todos los trabajadores, así como los requerimientos del entorno internoentorno. Los cambios básicos que se ha sufrido en la forma de vida y los prácticas cotidianas, condicionan un compás acelerado de la vida de uno, que genera ansiedad, debilitamiento emocional, perturbaciones en los ritmos de subsistencia, actividad física y descanso, con sufrimientos físicos-mentales y factores de peligro en la salud de los colaboradores del nuevo milenio (Apiquian, 2007).

Otros aspectos a considerar son los turnos laborales y el complicado horario de trabajo, el uso de transporte, las distancias geográficas, también generan stress máximo, así como la agregación de nuevas tecnologías en las organizaciones; las relaciones interpersonales y amicales, el salario, el mundo competitivo empresarial, de asunción a cargos superiores, etc.; son detonantes para una reacción en cadena de los trabajadores. Según algunas opiniones, otro aspecto que produce directamente el burnout es la estrategia empresarial de minimización de costos y maximización de trabajo, las tareas, los alargamientos de horarios de trabajo, así como en las que se reducen personal ampliando las funciones y responsabilidades de los trabajadores; se consideran detonantes para una vida tranquila.

Hacia 1986, Maslach y Jackson definieron elsíndrome de burnout desde una perspectiva tridimensional caracterizándola por:

a) Agotamiento emocional (cansancio y fatiga física, psíquica o como una combinación de ambos)

b) La despersonalización, el desarrollo de sentimientos, actitudes, y respuestas negativas, distantes y frías hacia otras personas, como los clientes, pacientes, usuarios, etc. Se acompaña de un incremento en la irritabilidad y una pérdida de motivación, es la sensación de no poder dar más de sí mismo a los demás. 
c) Sentimiento de bajo logro o realización profesional $\mathrm{y} / \mathrm{o}$ personal. Se presenta cuando las peticiones que se le hacen exceden su capacidad para atenderlas de forma competente. Supone respuestas negativas hacia uno mismo y hacia su trabajo, evitación de las relaciones personales y profesionales, bajo rendimiento laboral, incapacidad para soportar la presión y una baja autoestima.

Por lo que este síndrome, también es conocido como síndrome de aniquilación, síndrome de estar quemado, síndrome de desmoralización o síndrome de agotamiento emocional o profesional. Es considerado por la Organización Mundial de la Salud como una enfermedad laboral que provoca quebranto y pérdida de la salud física y mental de los individuos y por ende su deficiente productividad para las empresas.

Otro síndrome interesante es el síndrome de Mobbing, llamado también de acoso. Se presenta cuando una persona realiza una intimidación psicológica extrema, de forma consecuente, recurrente y durante un tiempo considerado para lograr que la otra persona o conjunto de personas no se sientan bien en el lugar de trabajo o con su oficio en particular; con el propósito de echar por tierra las redes de comunicación de la víctima o víctimas con otro colaborador, con el (jefe). Así como devastar su reputación, trastornar el ejercicio de sus labores y lograr que, finalmente, esa persona o personas acaben abandonando el lugar de trabajo, cansados de la manipulación y persecución que sufren.

Como vemos, son un conjunto de situaciones en las que el individuo se ve sometido y debe enfrentar dentro de las organizaciones, haciendo necesario prevenir y cortarlos si fuera posible.

\section{A partir del burnout al engagement: una nueva forma de hacer bien las cosas en las organizaciones resilientes}

Muchas veces se ha usado el termino el engagement, definido en el contexto laboral como "un estado psicológico positivo relacionado con el trabajo y que está caracterizado por vigor, dedicación y absorción o concentración en el trabajo" (Schaufeli et al., 2002, p.79),permitiendo y adaptando la definición de engagement como la construcción de relaciones estables y duradera que generen valor para la organización. Es una antítesis en el personal, el sentido de pertenencia, compromiso y lealtad. Un colaborador "enganchado" es aquel que no sentirá presionado, más por el contrario, serán aquellos en que normalmente reciben muy buenas referencias de jefes y compañeros. Este es uno de los grandes desafíos de la actualidad, gente comprometida que aplica planes estratégicos, generando entusiasmo, cumplimiento de objetivos o transmitiendo la idea de lo importante que es cada individuo dentro de los logros empresariales.

Es en este contexto, el engagement en las organizaciones resilientes se pueden puntualizar como aquellas que realizan esfuerzos ordenados, consecuentes y cuidadosos, planificados y proactivos para mejorar y superar todos los aspectos negativos que se vivió, en una de las etapas de la vida organizacional, principalmente, mediante buenas prácticas relacionadas con la mejora de los diseños de los puestos, mejora del ambiente laboral mediante una buena relación dirección y colaborador; estrategias y planes de acción permanentes y flexibles.

\section{¿Por qué mantener una actitud resiliente?}

El mantener una actitud resiliente mejora nuestro bienestar, en primer lugar, psíquico y por ende físico; es decir, mejora nuestro estado de ánimo y por ende nuestra salud, evadiendo problemas de estrés, ansiedad y todo el conjunto de males y síntomas que trae como consecuencia las crisis experimentadas.

La resiliencia como dijimos es un actitud ${ }^{7}$ una manera de estar dispuesto a comportarse u obrar (hacer frente a algo), derivada de un aprendizaje constante podemos entrenarla continuamente, aspirando cambiar los aspectos negativos de los reveses surgidos en aspectos positivos, proactivos y siendo más asertivos con nosotros mismos y con los demás

¿Cómo hacerlo? Sabemos que no podemos manejar el entorno, las circunstancias y si

7 RAE (s/f) Estado de la disposición nerviosa y mental, que se organiza a partir de las vivencias y que orienta o dirige la respuesta de un sujeto ante determinados acontecimientos 
no podemos cambiar las situaciones que nos rodean, pero sí podemos sustituir nuestra manera de pensar, nuestras actitudes. Esta forma de pensar es la que nos crea un sentimiento de bueno o malo, nos hace sentir mejor o peor; es ahí donde uno puede manejar la manera de cómo debes tomar esa situación, la que te golpeó la que te hizo sentir mal y pasar por momentos desagradables y cambiarlos por pensamientos más positivos, verlos desde otro ángulo, con un sentido de aprendizaje $\mathrm{y}$ de mejora personal u organizacional, inyectando esta dosis de positivismo en él y en los demás. De este modo, la organización se verá reconfortada y alimentada por gente proactiva.

Pérez y Gardey(2008) manifiestan que mediante,

"En la teoría de la consistencia cognitiva, las personas buscan la coherencia en su vida y que en base a conseguirla es que varían sus actitudes y pensamientos para sentir una unicidad en su ser interno pues la presencia de dos estados de consciencia (incoherencia) les incomoda. En este caso, la actitud tendría que ver con la sucesión de acciones que aseguren un equilibrio para el individuo"

Como podemos concluir es que el individuo/empresa al pasar por las distintas perturbaciones y crisis pierde el equilibrio en su vida y mediante esta teoría, se reconoce que el colaborador o la empresa tenga alguna conducta con la que recobrar el equilibrio, no solo emocional sino de fuerza y acción; trayendo consigo el mejoramiento y cambio de vida y de su perspectiva, no solo para la de él sino, también para la del grupo; es decir, la organización toda.

El pensar en que uno tiene la capacidad para afrontar una o muchas situaciones negativas y el entender que son parte de la vida, primero aceptando que nos tocó vivir esa experiencia y estar consiente que tendrá un proceso de malestar y sufrimiento y que luego tendremos que "pasar la hoja" de la vida, nos dará las fuerzas necesarias para seguir adelante.

\section{Algunas características de las organizaciones resilientes:}

Consideramos que existe un sinfín de características pero las que vamos a citar a continuación sugieren que son las más proactivas, y las citamos a continuación:

- Son exitosas e innovadoras

- Permanecen en el tiempo, se mantienen y/o crecen

- Capacidad de improvisación

- Disfrutan de una marca reconocida y gozan de una buena reputación

- Planifican su futuro a corto plazo

- Aprenden de manera constante

- Lideran y se anticipan a los cambios

- La cultura organizacional es dinámica, proactiva

- Trabajadores tienen alto sentido de pertenencia a la organización

- Capacidad para aprender y conectar los aprendizajes.

- Expresar expectativas positivas y realistas

- Los miembros de la organización tiene una marcada cultura de resiliencia.

- Confianza en sí misma/o, autonomía, flexibilidad.

- Compartir responsabilidades

Consideramos que estas son algunas de las muchas más que existe pero que por practicidad -aquello que es efectivo en el uso: que reporta un resultado válido y útil con el fin propuesto o el objetivo buscado ${ }^{8}$-, la citamos.

\section{Ejemplo de organizaciones resilientes:}

Teniendo en mente que las organizaciones deben estar preparadas para enfrentar una dificultad, acontecimiento crítico o una eventualidad y no tener una actitud de espera lenta, sino más bien recurren a tomar actitudes defensivas,

8 "Practicidad" (2009). En Significados.com. Disponible en: http://harquimedes.blogspot.pe/2009/10/practicidad.html 
protectoras o de contraataque cuando sea necesaria tal accionar. Es menester precisar el o las áreas que deben ser retroalimentas y reforzadas para cerciorarla permanencia en el mercado, el crecimiento constante tanto en mercado como en rentabilidad. Pero esto no es posible si las bases no son sólidas, es necesario analizar qué nivel de aceptación tiene la empresa en el entorno en que se desenvuelve. Debe de estar bien definido y precisado en un plan estratégico si la organización está dispuesta a plantear nuevas prácticas, correr riesgos, implantar nuevos procesos para solucionar los problemas y, si es preciso, erradicar los ya existentes por unos nuevos.

Existen empresas tan famosas y conocidas como Ford Motors, Microsoft, Toyota, etc. Pensamos y las relacionamos con la palabra exitosos, además de dar la impresión de ser empresas invulnerables que, por su fama, antigüedad, crecimiento mundial, numeroso grupo de personas que trabajan, activos, etc; son empresas maduras y fuertes que crecieron desafiando y venciendo todos los problemas que se les presentaron, soportando muchas dificultades en su camino hacia el triunfo.

Pero también tenemos a grandes empresas, solo para mencionarlas que han sido remecidos por las fuerzas de la competencia, las mismas que para no liquidar tuvieron que acogerse a la protección por bancarrota. Un ejemplo de ello es la gigante American Airlines en 2011, una de las líneas aéreas más grandes del mundo; así también tenemos el caso de la General Motors Corporation en 2009. León, F. (2015) citando a Justo, (2012, p.1.) Igualmente estos fenómenos también están afectando a los países europeos que, por razones como las guerras mundiales, sufren situaciones muy complicadas, como España que por su gran endeudamiento está atravesando una severa crisis. Las empresas mencionadas son un buen ejemplo de empuje y mucho esfuerzo y las que aún subsisten frente a todo el cumulo de experiencias vividas.

\section{CONCLUSIONES:}

Resiliencia organizacional no es un concepto, es una acción puesta en práctica en la empresa, y debe estar liderada por laalta dirección, para que trabajando en equipo se alcancen los objetivos organizacionales y se afronten adecuadamente las adversidades empresariales.

Las empresas resilientes no pueden predecir todas las amenazas de su entorno, pero si tienen la capacidad de planificar y realizar las acciones necesarias para minimizar los efectos de las amenazas, aprovechar las oportunidades y sobre todo fortalecer el clima organizacional.

La resiliencia organizacional permite romper paradigmas gerenciales que causan estrés laboral, desorganización, falta de compromiso, baja productividad. Logrando crear una nueva cultura empresarial, que permita optimizar el nivel de vida de los colaboradores, incrementar la productividad empresarial y satisfacer las insuficiencias y deseos de los clientes o interesados.

\section{REFERENCIAS BIBLIOGRÁFICAS:}

American Psychological Association, (1929) (estándares más reconocidos para la transmisión del conocimiento científico y académico).

Apiquian, A. (2007) Síndrome de burnout en las empresas. Coordinadora de Psicología Laboral Universidad Anáhuac. Presentación realizada en el Tercer Congreso de Escuelas de Psicología de las Universidades Red Anáhuac. Mérida, Yucatán. México Norte.

Arboix, M. (2014). Resiliencia, la capacidad de superar las adversidades.

Barrajón, L (2012) UBUNTU: Una palabra puede significar mucho.

Becoña, E. (2006) Resiliencia: Definición, características y utilidad del concepto. Facultad de Psicologia de Santiago de Compostela.

Chávez, E. y Yturralde, E. (2006) citado en: http://es.wikipedia.org/wiki/Resiliencia_ (psicolog\%C3\%ADa).

Clegg, S., / Porras, S. (2007). Encontrando sentido a la globalización en la teoría de la organización; ¿Negocios globales? Redes Organizacionales. México: UAM - Iztapalapa.

FH: Fundación Factor Humá. (2010). Unidad de conocimiento - La Resiliencia en las organizaciones.

Flores, A. / Grané, J. (2008): La resiliencia. Crecer desde la adversidad. Barcelona: Plataforma Editorial. 
González, J. (2002) El maltrato psicológico: Cómo defenderse del mobbing y otras formas de acoso.

Grotberg, E. (2001). Nuevas tendencias en resiliencia.

ICCB, Instituteon Child Resilience and Family (1994) Resiliencia: ¿Proceso de superación de la adversidad?

Jericó, P. (2009). Personas y Empresas.

Kotliarenco, M. / Cáceres, I. / Álvarez, E. (1996). Resiliencia: Construyendo en adversidad. CEANIM, Santiago, Chile.

León, F. (2015) La Resiliencia: Su Aplicación en el Sector Empresarial. Revista: CE Contribuciones a la Economía. Universidad Ecotec.

León, P. (2013). RESILIENCIA ORGANIZACIONAL: Una aproximación. Bogotá, Colombia: Facultad de Administración Universidad del Rosario.

Leymann. H. (1990) Mobbing o acoso psicológico. Universidad de Estocolmo área de Psicología del Trabajo. Congreso sobre Higiene y Seguridad en el Trabajo.

Luthard, S. / Cicchetti, D. (2000). The construct of resilience: Implications for interventions and social policies. Developmental and Psychopathology, 12, 857-885.

Maslach, C. / Jackson, S. (1986): The Maslach Burnout Inventory. (Zaed). Palo Alto, ConsultingPsychologistsPress (Versión Española adaptada por Nicolás Seisdedos Cubero, TEA 1987)

Medina, C. (2011). La resiliencia y su empleo en las organizaciones. Gestión y estrategia \#41, 41.

Medina, C. (2012). La resiliencia y su empleo en las organizaciones. Gestión y estrategia

Nemeth, C. / Hollnagel, E. / Dekker, S. (Eds.) (2009). Resilience Engineering Perspectives, Volume 2: Preparation and Restoration. Farnham, UK: Ashgate.

Oszlak, O. (2003) ¿Responsabilización o respondibilidad?: El sujeto y el objeto de un estado responsable. VIII Congreso Internacional del CLAD sobre la Reforma del Estado y de la Administración Pública, Panamá.

Pérez, J. / Gardey, A. (2008) Definición de relaciones interpersonales.

Pérez, J. / Gardey, A. (2013) La Resiliencia.

Ramos, R. (2014) La importancia de la resiliencia.
Ramírez, P. / Hernández, E. (2012). Resiliencia familiar, depresión y ansiedad en adolescentes en situación de pobreza. Revista de Enfermería del Instituto Mexicano del Seguro Social.

Rutter, M (1981) Maternal DeprivationReassessed, Secondedition, Harmondsworth, Penguin.

Rutter, M. (2012). Resilience: Casual pathways and social ecology.

Schaufeli, W. / Salanova, M. / González-Romá, V. y Bakker, A. (2002). The measurement of burnout and engagement: A confirmatory factor analytic approach. Journal of HappinessStudies, 3, 7192.

Talavera, B. / Luceño, L. / García, J. / López, Maㅗ I. (2012) La resiliencia en las empresas: ¿de qué estamos hablando? Laboratorio de Psicología del Trabajo y Estudios de Seguridad de la Universidad Complutense de Madrid (UCM).

Vanistendael, S. (1994) La Resiliencia: un concepto largo tiempo ignorado. En Revista: La infancia en el mundo. Vol 5, $\mathrm{N}^{\circ}$ 3. BICE. Montevideo Uruguay.

\section{Otras literatura consultada:}

Carrasco, A. / De la Corte, C. / León, J. (2010) Engagement: A Resourceto0ptimizeThePsychosocialHealth In Organizations And PreventBurnout And Job Stress (Engagement: Un recurso para optimizar la salud psicosocial en las organizaciones y prevenir el burnout y estrés laboral).

Condia, H. (2011). Manejo de las Crisis Personales y Profesionales desde el Enfoque de la Resiliencia. Bogotá, Colombia: Universidad del Rosario.

Cruz, O. / Ovalle, M., / Pando, M. (2008). Mobbing y estrés en académicos de la Diversidad de Ciencias y Artes de Chiapas. México

Herrera, G. / Rodríguez, G. (2016) Turismo y sistemas empresariales resilientes: Factores Críticos de adaptabilidad en baños de agua santa - Ecuador.

Hollangel, E. (2011). Resilience Engineering in Practice: A Guide. England: Ashgates Publishing Limited.

Melillo, A. / Suarez, E. (2001) Resiliencia. Descubriendo las propias fortalezas. Buenos Aires: Paidós.

Navarrete, S. (2010). Mobbing en México. Apuntes para su fundamentación. Mobbing: ¿Debemos seguir llamándolo así? Lorenz y Leymann revisitados. $11^{\circ}$ Congreso Virtual de Psiquiatría Interpsiquis 2010. 
Peña, F. / Ravelo, P. / Sánchez, S. (2007). Cuando el trabajo nos castiga. Debates sobre el mobbing en México. México: Ediciones y Gráficos Eón, S. A. de C. V.

Pereira, R. (2010). Trabajando con los recursos de la familia: Factores de resiliencia familiar. Sistemas familiares.

Rodríguez, F. (2016). Concepto de Resiliencia y Resiliencia Organizacional.
Romero, D. (2016) ¿Qué es el engagement y cómo lo puedes aplicar a tu empresa?

Ungar, M. (2015). Practitioner Review: Diagnosing childhood resilience: A systemic approach to the diagnosis of adaptation in adverse social ecologies. Journal of ChildPsychology and Psychiatry. 